\title{
Cystine addiction of triple negative breast cancer associated with EMT augmented death signaling
}

\author{
Xiaohu Tang ${ }^{1,2}$, Chien-Kuang Ding ${ }^{1,2}$, Jianli $\mathbf{W u}^{1,2}$, Jessie Sjol ${ }^{3}$, Suzanne Wardell ${ }^{4}$, Daniel \\ George $^{5}$, Donald P. McDonnell ${ }^{4}$, David S. Hsu ${ }^{2,4}$, Jeffrey T Chang ${ }^{3}$, and Jen-Tsan Chi $^{1,2}$ \\ ${ }^{1}$ Department of Molecular Genetics and Microbiology, Duke University, Durham, North Carolina \\ 27710 \\ ${ }^{2}$ Center for Genomic and Computational Biology, Duke University, Durham, North Carolina 27710 \\ ${ }^{3}$ Department of Integrative Biology and Pharmacology, McGovern Medical School, The University \\ of Texas Health Science Center at Houston, Houston, Texas 77030 \\ ${ }^{4}$ Department of Pharmacology and Cancer Biology, Duke University, Durham, North Carolina \\ 27710 \\ ${ }^{5}$ Department of Medicine, Duke University, Durham, North Carolina 27710
}

\section{Abstract}

Despite the advances in the diagnosis and treatment of breast cancer, breast cancers still cause significant mortality. For some patients, especially those with triple negative breast cancer (TNBC), current treatments continue to be limited and ineffective. Therefore, there remains an unmet need for a novel therapeutic approach. One potential strategy is to target the altered metabolic state that is rewired by oncogenic transformation. Specifically, this rewiring may render certain outside nutrients indispensable. To identify such a nutrient, we performed a nutrigenetic screen by removing individual amino acids to identify possible addictions across a panel of breast cancer cells. This screen revealed that cystine deprivation triggered rapid programmed necrosis, but not apoptosis, in the basal-type breast cancer cells mostly seen in TNBC tumors. In contrast, luminal-type breast cancer cells are cystine-independent and exhibit little death during cystine deprivation. The cystine addiction phenotype is associated with a higher level of cystinedeprivation signatures noted in the basal type breast cancer cells and tumors. We found that the cystine-addicted breast cancer cells and tumors have strong activation of TNFa and MEKK4-p38Noxa pathways that render them susceptible to cystine deprivation-induced necrosis. Consistent with this model, silencing of TNFa and MEKK4 dramatically reduces cystine-deprived death. In addition, the cystine addiction phenotype can be abrogated in the cystine-addictive cells by miR-200c, which converts the mesenchymal-like cells to adopt epithelial features. Conversely, the introduction of inducers of epithelial-mesenchymal transition (EMT) in cystine-independent breast cancer cells conferred the cystine-addiction phenotype by modulating the signaling components of cystine addiction. Together, our data reveal that cystine-addiction is associated with EMT in breast

Users may view, print, copy, and download text and data-mine the content in such documents, for the purposes of academic research, subject always to the full Conditions of use: http://www.nature.com/authors/editorial_policies/license.html\#terms

Conflict of interest

The authors declare no conflict of interests. 
cancer during tumor progression. These findings provide the genetic and mechanistic basis to explain how cystine deprivation triggers necrosis by activating pre-existing oncogenic pathways in cystine-addicted TNBC with prominent mesenchymal features.

\section{Introduction}

Treatment for breast cancer has advanced significantly over the past decades and is partly responsible for the recent improvement in disease outcome ${ }^{1}$. Unfortunately, even with these advances, the American Cancer Society estimates that 40,290 US women will have died of the disease in 2015. Clearly, a deeper understanding of the biology underlying the cancer phenotype is still needed. To address this need, genomic analyses have revealed the genetic dysregulation seen across cancers ${ }^{2}$. However, few effective therapeutic targets have yet emerged from these intensive efforts. Alternatively, oncogenic progression is often associated with dysregulation of basic metabolic processes. Because this rewiring of cancer metabolism may lead to new vulnerabilities, a detailed knowledge of cancer biochemistry may be of equal or greater importance in the understanding and treatment of the disease ${ }^{3-6}$.

While untransformed cells can adapt to a wide range of metabolic conditions, such flexibility may become limited during oncogenic transformation and under the stress conditions commonly seen in solid tumors ${ }^{7}$. One example is the Warburg effect, a central concept of tumor physiology where the dependence on glycolysis for energy renders cancer cells addicted to glucose and sensitive to inhibition by glucose analogues 8,9 . More recently, we found that basal-like breast cells that exhibit a robust hypoxia program also have a dysregulated and inflexible metabolic program ${ }^{10}$. Similarly, oncogenic mutations can also limit metabolic flexibility by altering the requirements for amino acids. The best characterized requirement is glutamine, which is reported to be essential for cancer cells harboring a range of oncogenic events including c-myc activation ${ }^{11-13}$, inhibition of Aktmediated glycolysis ${ }^{14}$ and IDH1 mutation ${ }^{15}$, as well as in basal-type breast cancer cells ${ }^{16}$. In addition, other amino acids have been reported to be indispensable in some tumors, such as leucine ${ }^{17}$, arginine ${ }^{18}$, methionine ${ }^{19}$ and valine ${ }^{20}$. Taken together, this wealth of data indicates that amino acid addiction is a common phenomenon that varies significantly among normal and cancerous cells, which presents a potential therapeutic window.

To guide therapy, breast cancers are currently categorized by histology, receptor status, and intrinsic subtype (which broadly overlaps with receptor status). Furthermore, the metabolic phenotypes and heterogeneity of breast cancers have been defined based on the global metabolomic profiling ${ }^{6,21}$. In general, however, the nutritional requirements have not yet been mapped backed to these established descriptors of the disease. Two exceptions are found in basal-type breast cancer cells, which have been found to be glutamineaddicted ${ }^{1622}$, and are also sensitive to XCT (SLC7A11) inhibitors that block cystine uptake ${ }^{22}$. This suggests that they are addicted to cystine, but the underlying mechanism for the cell-type specificity in basal-type breast cancer cells remains unknown.

Here, we performed a nutrigenetic screen on a panel of breast cancer cells by removing single nutrient components and determining the resultant cellular and gene expression phenotypes. A similar approach has identified the leucine addiction of melanoma cells ${ }^{17}$, 
epigenetic regulation by methionine ${ }^{23}$ and profound cystine addiction in VHL-deficient RCC cells ${ }^{24}$. Based on our study, we identified a similar cystine addiction specifically in the basal-type breast cancer cells that are found in triple negative tumor cells. Furthermore, we have found that these cystine-addicted basal cells exhibit a signaling program that sensitizes them to activation of canonical programmed necrosis pathways. Introduction of several regulators of EMT modulates the apparently basal-specific stress-activated signaling. These studies illustrate that cystine deprivation strategies may target the most problematic mesenchymal-like breast cancer cells, which are known to have increased stem cell properties and chemo-resistance.

\section{Results}

\section{Nutrigenetic screens of a panel of breast cancer cells}

Previously we established a nutrigenetic screen by removing one (or all) amino acids from culture media. This made possible the association of nutrient addiction with particular tumor subtypes by determining the resultant gene expression or cellular phenotypes across a panel of genetically defined cells ${ }^{23,24}$. We then applied this strategy to six breast cell lines representing the luminal (BT474, ZR751, MCF7) and basal (MDA-MB-157, BT20, MDAMB-231) subtypes ${ }^{25}$. These cells were subjected to deprivation of each of 15 amino acids in DMEM (supplemented with dialyzed FBS) at a time to evaluate their effect on viability. We found that the deprivation of most individual amino acids for 48 hours arrested cell proliferation without causing significant death. Unexpectedly, the deprivation of cystine, the dimeric and predominant form of cysteine in media, induced acute and extensive cell death in the three basal, but not the luminal cancer cells (Fig 1A). This cystine-deprived death was validated by crystal violet staining (Fig 1B) under low $(1 \mu \mathrm{M})$ cystine level. To comprehensively evaluate cysteine addiction in breast cancer, we further performed cysteine deprivation on nine more breast cancer cell lines, which represent all major subtypes of breast cancer (luminal/ERBB2, Basal A, and Basal B types). Collectively, these data indicated that $5 / 6$ basal B breast cancer cells (with the exception of MDA-MB-436) were highly sensitive to cysteine depletion (Fig S1A and S1B). In contrast, most basal-A cells (3/4, with the exception of BT20) and all luminal/ERBB2 cells (7/7 cell lines) exhibited resistance to cysteine deprivation.

The phenotypic features of cystine-deprived cell death in basal-B breast cancer cells were highly similar to cystine-deprived necrosis previously characterized in $V H L$-null RCC cells ${ }^{24}$. Canonical programmed necrosis ${ }^{26}$ is induced by a signaling cascade consisting of RIPK1 (Receptor-interacting kinase 1), RIPK3 (Receptor-interacting kinase 3) and MLKL (Mixed Lineage Kinase domain-Like). We found that the RIPK1 inhibitor necrostatin-1 (Nec-1), the MLKL phosphorylation blocker necrosulfonamide (NSA), and the mitochondria reactive oxygen species (ROS) scavenger Necrox-5 (Nec-5) all significantly rescued cystine-deprived death (Fig 1C). In contrast, we saw no evidence of apoptosis as the pan-caspase inhibitor Z-Vad-FMK (Z-Vad) did not rescue the cystine-deprived cell death phenotype (Fig 1C). Further supporting a specific role for necrosis, cystine deprivation of MDA-MB-231 led to a large increase in a propidium iodide (PI) positive population, with no change in annexin- $\mathrm{V}$ (Figure S1C). In a luminal cell, MCF-7, cysteine deprivation resulted 
in only a small increase of PI positive and annexin- $\mathrm{V}$ positive populations. Thus, cystinedeprivation triggers cell death in basal breast cancer cells through the induction of RIPK1/3MLKL-dependent canonical necrotic death.

Having seen a higher requirement for cystine in basal cells, one possible explanation may be related to the fact that cystine is a major source for glutathione synthesis. And, the importance of glutathione in the context of cysteine deprivation has been previously observed since glutathione supplement is sufficient to rescue cysteine-deprived necrosis and blocking of glutathione synthesis by L-buthionine sulfoximine mimics cysteine-deprived necrosis in kidney cancer cells ${ }^{24}$. To test whether glutathione synthesis is more dependent upon exogenous cystine in basal-like breast cells, we treated MDA-MB-231 and MCF7 cells with different concentrations of cystine. This revealed that GSH and GSSG levels dropped dramatically to undetectable levels only in the cystine-addicted MDA-MB-231 cells (Fig S1D). Further, with cystine deprivation, the GSSG/GSH ratio increased much more significantly in MDA-MB-231 cells than MCF7 (Fig S1D). These results suggest that MDAMB-231 cells are more dependent upon external cystine to maintain redox balance.

\section{TNFa is required for cystine-deprived necrosis in basal-type breast cancer cells}

To determine the pathways driving basal-type specific cystine addiction, we determined the pathway activities of breast cancer cell lines using a panel of gene expression signatures derived from in vitro perturbation ${ }^{10,27}$. This analysis revealed that basal-type breast cancer cells have a significantly higher level of TNFa activation, concomitant with low levels of ER, PR and ERBB2 (Fig 2A), consistent with the low receptor levels seen in basal-type or TNBC cells. The elevated TNFa is consistent with our prior study showing a role for TNFa in cystine addiction in renal cell carcinoma ${ }^{24}$. Therefore, we tested the involvement of $\mathrm{TNFa}$ in basal-like breast cancer by silencing it using two independent shRNAs. The TNFa-silenced cells had reduced cystine-deprived necrosis (released proteases, Fig $2 \mathrm{~B}$ and S2A) and increased viability (crystal violet, Fig 2C and S2B). These data indicate that high $\mathrm{TNFa}$ activity in basal-type cancer cells is essential for cystine-deprived necrosis.

\section{Elevated levels of MEKK4-p38-Noxa pathway in basal type breast cancer cells contribute to cystine-deprived necrosis}

In VHL-deficient RCC cells, cystine-deprived necrosis depends on a p38 (MAPK14) - Noxa (PMAIP1) pathway ${ }^{24}$. To determine whether a similar mechanism is involved in basal-type breast cancer, we silenced TNFa and measured the impact on the p38-Noxa pathway under high $(200 \mu \mathrm{M})$ and low $(1 \mu \mathrm{M})$ cystine conditions (Fig 2D). Interestingly, we find that TNFa silencing reduced the $\mathrm{p} 38$ phosphorylation and Noxa induction that was triggered by cystine deprivation (Fig 2D and S2C). p38 phosphorylation and Noxa protein were strongly induced by cystine deprivation in the basal-type cells, but not in luminal-type cells (Fig S2D and S2E). Using different MAPK inhibitors, we confirmed that the p38 inhibitors (SB-203580 and SB202190), but not JNK inhibitors (SP-600125 and C401), protected cells from the cystine-deprived death (Fig S2F and S2G). Silencing of Noxa by shRNA also caused the basal cells to become resistant to cystine-deprived necrosis (Fig S2G). These results demonstrate that the activation of the p38-Noxa pathway is critical for cystine-deprived 
necrosis in basal-type breast cancer cells, analogous to the mechanism we reported in VHLnull $\mathrm{RCC}^{24}$.

Given the importance of $\mathrm{p} 38$, we looked for activation of relevant upstream MAPK pathways across breast cancer cells. p38 MAPK is activated by MEKK4 (MAP3K4), a major mediator of environmental stresses, via phosphorylation of MKK3/6 ${ }^{28}$. MEKK4 itself can be activated by RACK1 (GNB2L1) or GADD45A ${ }^{29}$. With cystine deprivation, NOXA and GADD45A were further induced in basal cells, but not in luminal cells (Fig S2D and S2E). Interestingly, when compared with luminal cells, basal type breast cells had higher baseline levels of almost all components of MEKK4 pathway (MEKK4, RACK1, p38 and NOXA) (Fig 3A), even before cystine deprivation. The mRNA levels of RACK1 and GADD45A were also increased in basal breast cells (Fig S3A). However, MEKK4 mRNA expression was not increased, as seen with the protein levels, suggesting additional posttranscriptional regulation (Figure S3A).

Given the increased expression of the MEKK4 pathway, we examined the role of MEKK4 in the signaling of cystine-deprived necrosis. Silencing MEKK4 by shRNAs significantly protected BT549, MDA-MB-231 and MDA-MB-157 from cystine-induced necrosis (Fig 3B, S3B and S3C) and reduced the phosphorylation of MKK3/6 and p38 (Fig 3C). Taken together, these data suggest that the activation of the MEKK4-p38-Noxa pathway by cystine deprivation is essential for cystine-deprived necrosis. The elevation of nearly all components in this pathway in basal-type breast cancer cells may associate with and contribute to the cystine addiction.

\section{Correlation between cystine-deprivation and TNFa signatures in breast cancer cells and tumors}

Previously, we found that the cell signaling and subsequent transcription responses triggered by cystine deprivation, but not the metabolic changes, are determinants for the type of cell death in RCC tumor cells ${ }^{24}$. We defined a cystine-deprivation gene expression signature (CysDep-sig) based on the transcriptional changes mediated by cystine deprivation in $V H L$ null RCC cells ${ }^{24}$. This gene expression signature (like others) represents a quantitative phenotype and serves as a marker of a biological activity. Comparing the similarity between a gene expression signature and transcriptional profile from a sample of interest, such as an experimental perturbation or a tumor sample, allowed us to predict the activation of the signature in the sample. Similar gene signature approaches have been used to define the influences of oncogenic signaling and TME stresses in multiple cancer types 10, 30, 31 . When this cystine-deprivation signature was projected to a panel of breast cancer cells, we found that the basal type breast cancer cells had much higher level of CysDep-sig than the luminal cancer cells (Fig 4A). In addition, CysDep-sig was also positively correlated with a TNFa signature, despite the fact that they shared very few common probesets (Fig 4B). These strong associations were consistent with the cystine-addicted phenotype seen in the basaltype cancer cells. In breast tumors (GSE69031) ${ }^{32}$, we found that the cystine deprivation signature was significantly enriched in the ER- breast tumors, compared with ER+ ones (Fig 4C). It was also significantly higher in basal subtype tumors (Fig 4D), and positively correlated with the TNFa signature (Fig 4E). Importantly, we found that a strong cystine- 
deprived signature was associated with relatively poor clinical outcome (Fig 4F). These results suggest that basal type breast tumors, enriched in both TNFa and cystine-deprived signatures, may also be characterized by cystine addiction. Both TNFa and cystine-deprived signatures may be used to predict the sensitivity to cystine starvation and cystine addiction phenotypes.

\section{EMT modulates cystine-deprived signaling and contributes to the cystine addiction phenotype of basal type breast cancer cells}

One common feature of basal type breast cells and tumors is their prominent mesenchymal features ${ }^{33-35}$. To determine the potential association between mesenchymal features and cystine addiction, we performed a series of experiments to alter the mesenchymal or epithelial state in isogenic cell line models, and determined their effects on cystine addiction and independence. The miR-200 family miRNAs, which are frequently lost in basal-type breast tumor cells, mediate the mesenchymal to epithelial transition. Therefore, we introduced miR-200c into the cystine-addicted MDA-MB-231 cells with prominent mesenchymal feature. As expected, the introduction of miR-200c (Fig S4A) dramatically increased the expression of E-cadherin (Fig 5A) and reduced Zeb2 (Fig 5A), suggesting its conversion to an epithelial state. Importantly, miR-200c also reduced cystine-deprived necrosis (Fig 5B). We found that miR-200c expression reduced the protein expression of MEKK4 and RACK1 (Fig 5C). The mRNA expression of MEKK4 was not decreased as significantly, potentially due to post-transcriptional regulation noted above in the luminal and basal breast tumor cells (Fig 3A and S3A). These results indicate that the cystine addiction phenotype is a characteristic of the mesenchymal state that is inhibited by miR-200c.

Next, we tested whether the conversion to a mesenchymal fate will confer a cystine addiction phenotype. To test this possibility, we introduced two EMT inducers, Twist (TWIST1) and Snail (SNAI1), into cystine-independent luminal breast cells T47D ${ }^{36}$. Both EMT inducers triggered mesenchymal features, including reduced E-cadherin/claudin-1 expression and increased vimentin (Fig S4B and S4C). Interestingly, both Twist and Snail induced high p38 phosphorylation and Noxa protein expression in T47D cells (Fig 5D). Importantly, we found that cystine deprivation triggered massive necrosis in both Twist- and Snail-expressing T47D cells (Fig 5E). This shows that EMT suppressor or inducers modulate signaling pathways that render cell sensitivity to cysteine deprivation. Our data supported the idea that the cell-type specific cystine addiction phenotype is associated with the mesenchymal features in breast cancer cells.

Collectively, our data allow us to propose a novel model of cell-type specific cystine addiction associated with EMT in breast cancer cells (Fig 6). The cystine-deprived cell necrosis consists two phases - initiation and execution phases. The initiation phase includes MEKK4, p38 and Noxa that are required for signaling cascades of cystine deprivation induced necrosis. The execution phase comprises activation of the TNFa-RIPK1/RIPK3/ MLKL pathway involved in canonical programmed necrosis. Cystine-addicted cells predispose higher baseline levels of several signaling components of the initiation and execution phase of cystine-deprived necrosis, including elevated levels of components of the 
MEKK4/p38/Noxa pathway and higher activity of TNFa and cystine-deprived gene signatures. Importantly, we found that the EMT process contributes to the changes of signaling components in cystine-deprived necrosis and confers cells with a cystine addiction phenotype. Thus, cystine addiction may present a novel form of nutrient addiction in the basal-type breast cancer associated with EMT.

\section{Discussion}

While cystine/cysteine is appreciated as a limiting component of glutathione (GSH) synthesis that can be therapeutically targeted, delivery of the therapeutics is currently difficult due to a limited knowledge of the mechanism underlying cystine addition, and attendant lack of biomarkers that identify those cystine-addicted tumors. In this study, we have uncovered a specific cystine addiction in basal-B type breast cancer cells. This observation was consistent with the higher level of $\mathrm{xCT}$ and sensitivities to $\mathrm{xCT}$ inhibitors in basal-B type of breast cancer cells reported by others ${ }^{22}$. However, our study provided additional mechanistic insights into the regulatory mechanisms underlying basal-B type specific cystine addictions. We found that cystine-addicted cells have higher pre-existing levels of TNFa activity, EMT and multiple signaling components that play a role in the programmed necrosis triggered by cystine deprivation. Specifically, they have higher mRNA and/or protein levels of multiple components in the MEKK4-p38-Noxa pathway. In these cystine-addicted cells, cystine deprivation triggers the activation of this pathway and further primes the TNFa-RIPK1/RIPK3-MLKL necrosis execution pathway. Conversely, in cystineindependent cells, these pathways are not activated by cystine deprivation, and significant cell death is absent.

TNFa is able to induce EMT in many different types of tumors, and its activity frequently increases with EMT progression ${ }^{37,38}$. EMT in breast cancers contributes significantly to tumor progression by affecting proliferation, migration, invasion and angiogenesis 39,40 . However, our results indicate that the dysregulated signaling associated with EMT may also bring about a novel metabolic vulnerability that can be therapeutically exploited. We found that expression of EMT regulators, miR-200c, Twist and Snail, changes the signaling components of cystine-deprived necrosis and cystine addiction phenotype in breast tumor cells. EMT inducers lead to an increase in the baseline levels of these proteins, sensitizing cells to necrosis even before cystine deprivation. Overexpression of EMT inducers in luminal type cells increases levels of Noxa and activated p38, and confers the cystine addiction phenotype. Similar upregulation of $\mathrm{p} 38$ phosphorylation associated with mesenchymal transition was also found in prostate cancer ${ }^{41}$. Interestingly, CD44, highly expressed in basal-type breast cancer cells, can associate with and stabilize cystine transporter $\mathrm{xCT}$ to enhance the import of cystine and predict sensitivity to the $\mathrm{xCT}$ inhibitor sulfasalazine ${ }^{42,43}$. These data strongly suggest that cystine addiction is a cell type specific feature mostly associated with mesenchymal-type tumor cells in breast cancers. However, the underlying mechanisms by which these EMT regulators control each of these components remain incompletely understood. These will be the focus of our future research efforts. 
Based on our findings, we propose that cystine addicted cells may rely on continuous cystine import and de novo GSH synthesis to protect from higher levels of oxidative stress. Consistent with this idea, a recent metabolomic analysis of TCGA breast tumors found higher GSH levels in basal-like breast tumors ${ }^{6}$. Therefore, such convergence of cystine addiction and tumor progression may open a significant therapeutic window for basal-type breast cancers for which effective treatments are urgently needed.

Our discovery of cell-type specific cystine addiction may present therapeutic opportunities to selectively target clinically challenging tumors based on such mechanistic insights 44,45 . While sulfasalazine has been shown to reduce tumor growth and distant metastases in xenograft models of head/neck and breast cancer $22,43,46$, our studies provide additional novel insights. First, cystine addiction is found mostly in basal-B type breast cancer with a significant therapeutic window. Second, since cystine deprivation triggers programmed necrosis instead of apoptosis, it may bypass many chemo-resistant mechanisms that generally avoid apoptotic pathways. Last, high GSH levels are usually associated with chemoresistance ${ }^{47}$. Therefore, cystine deprivation may deplete intracellular GSH and overcome chemoresistance. However, for clinical translation, it will be important to further elucidate the intrinsic addiction mechanism and define predictive biomarkers to identify patient populations sensitive to cystine-targeted therapies.

\section{Materials and Methods}

\section{Materials, tissue culture and amino acid-deprivation protocol}

Reagents were obtained from the following sources: Cell culture grade amino acids from Sigma; $z$-VAD-FMK, Necrostatin-1, Necrosulfonamide from Calbiochem; Necrox-5 from Santa Cruz Biotechnology Inc; Antibodies to phospho-p38, total p38, $\beta$-tubulin, claudin-1 and Vimentin (D21H3) from Cell Signaling Technology; RACK1 antibody from BD; Antibodies to TNFa and MEKK4 from Abcam; Noxa antibody from Novus Biologicals.

All breast tumor cells were cultured in DMEM with $10 \%$ heat-inactivated FBS, $1 \%$ penicillin-streptomycin in a humidified incubator at $37^{\circ} \mathrm{C}$ and $5 \% \mathrm{CO}_{2}$. To prepare media deficient in each amino acid, Earle's balanced salt solution was supplemented with $4.5 \mathrm{~g} / \mathrm{L}$ glucose, $0.37 \mathrm{mM}$ sodium bicarbonate, $24.8 \mu \mathrm{M}$ ferric nitrates, 10\% dialyzed FBS (Sigma) and MEM vitamin solution (Invitrogen). Different amino acid combinations were then added. Cells were plated in the complete DMEM media 1 day prior to PBS-rinsing and amino acid-deprivation.

\section{GSH and GSSG measurement}

The GSH and GSSG were measured by LC-MS/MS according to a previous published method ${ }^{48}$ using GSH-NEM (N-ethylmaleimide)-D ${ }^{3}$ and GSSG-D ${ }^{6}$ as internal standard. The measurement of GSH (as GSH-NEM derivative) and GSSG was performed on Shimadzu 20A series liquid chromatography (LC) and Applied Biosystems/SCIEX API5500 QTrap tandem-mass spectrometer(MS/MS). MS/MS conditions: MRM transitions (m/z) followed for GSH-NEM, GSH-NEM-d3, GSSG, and GSSG-d6 were 433-304, 436.2-307.1, 613.1-355, and 619.1-361, respectively. Calibration samples for GSH and GSSG were 
prepared and analyzed alongside the study samples. Quantification was performed by Analyst 1.6.2 software.

\section{Cell cytotoxicity and survival}

Cell death induced by cystine deprivation was measured by CytoTox-Fluor ${ }^{\mathrm{TM}}$ Cytotoxicity Assay (Promega), cell number (crystal violet staining, counting) or CellTiter-Glo® Assay (Promega)).

\section{Western blot analysis}

Cells were lysed in RIPA buffer (Sigma) supplemented with protease inhibitor and PhosSTOP phosphatase inhibitor cocktail (Roche). Protein concentrations were determined by BCA protein assay. Equal amounts of protein were loaded for the immunoblot analyses. The signal was detected by the ECL plus Western blotting detection system (Amersham).

\section{RNA isolation and Real-time RT-PCR}

RNA was extracted by RNeasy kit (Qiagen), and total RNA was reverse-transcribed to cDNA using SuperScript-II reverse transcriptase (Life Technologies) and random hexamers. The level of gene expression was measured by quantitative PCR (qPCR) with Power SYBR Green PCR Mix (Applied Biosystems). All primers were listed in Supplementary Table S1.

\section{Gene signature projection analyses}

Cystine deprivation (CysDep) ${ }^{24}$, TNFa ${ }^{10}$ gene signatures were derived from previous reports. The projection analysis of these signatures to a breast tumor dataset (GSE69031) was performed according to our previous reports $10,31,49$.

\section{Statistical Analyses}

Experimental results were analyzed with a Student's two-sided t test using Prism (GraphPad). Data were expressed as mean \pm SD and a $\mathrm{p}$ value $<0.05$ was considered statistically significant.

\section{Supplementary Material}

Refer to Web version on PubMed Central for supplementary material.

\section{Acknowledgments}

We wish to acknowledge the financial support from the NIH (CA125618 and CA106520 to JTC) and Department of Defense (W81XWH-12-1-0148, W81XWH-14-1-0309, W81XWH-15-1-0486 to JTC). We appreciate the technical assistance of Ivan Spasojevic and the DCI's Pharmacokinetics and Investigational Chemotherapy share facility. We are also grateful to members of Chi lab for critical discussions and editing of the manuscript.

\section{References}

1. Berry DA, Cronin KA, Plevritis SK, Fryback DG, Clarke L, Zelen M, Mandelblatt JS, Yakovlev AY, Habbema JD, Feuer EJ. Cancer I, Surveillance Modeling Network C. Effect of screening and adjuvant therapy on mortality from breast cancer. N Engl J Med. 2005; 353:1784-1792. [PubMed: 16251534] 
2. Cancer Genome Atlas N. Comprehensive molecular portraits of human breast tumours. Nature. 2012; 490:61-70. [PubMed: 23000897]

3. Sreekumar A, Poisson LM, Rajendiran TM, Khan AP, Cao Q, Yu J, Laxman B, Mehra R, Lonigro RJ, Li Y, Nyati MK, Ahsan A, Kalyana-Sundaram S, Han B, Cao X, Byun J, Omenn GS, Ghosh D, Pennathur S, Alexander DC, Berger A, Shuster JR, Wei JT, Varambally S, Beecher C, Chinnaiyan AM. Metabolomic profiles delineate potential role for sarcosine in prostate cancer progression. Nature. 2009; 457:910-914. [PubMed: 19212411]

4. Dang L, White DW, Gross S, Bennett BD, Bittinger MA, Driggers EM, Fantin VR, Jang HG, Jin S, Keenan MC, Marks KM, Prins RM, Ward PS, Yen KE, Liau LM, Rabinowitz JD, Cantley LC, Thompson CB, Vander Heiden MG, Su SM. Cancer-associated IDH1 mutations produce 2hydroxyglutarate. Nature. 2010; 465:966. [PubMed: 20559394]

5. Hakimi AA, Reznik E, Lee CH, Creighton CJ, Brannon AR, Luna A, Aksoy BA, Liu EM, Shen R, Lee W, Chen Y, Stirdivant SM, Russo P, Chen YB, Tickoo SK, Reuter VE, Cheng EH, Sander C, Hsieh JJ. An Integrated Metabolic Atlas of Clear Cell Renal Cell Carcinoma. Cancer Cell. 2016; 29:104-116. [PubMed: 26766592]

6. Tang X, Lin CC, Spasojevic I, Iversen E, Chi JT, Marks JR. A joint analysis of metabolomics and genetics of breast cancer. Breast Cancer Res. 2014; 16:415. [PubMed: 25091696]

7. Keenan MM, Chi JT. Alternative fuels for cancer cells. Cancer J. 2015; 21:49-55. [PubMed: 25815843]

8. Song CW, Clement JJ, Levitt SH. Preferential cytotoxicity of 5-thio-D-glucose against hypoxic tumor cells. J Natl Cancer Inst. 1976; 57:603-605. [PubMed: 824455]

9. Lampidis TJ, Kurtoglu M, Maher JC, Liu H, Krishan A, Sheft V, Szymanski S, Fokt I, Rudnicki WR, Ginalski K, Lesyng B, Priebe W. Efficacy of 2-halogen substituted D-glucose analogs in blocking glycolysis and killing "hypoxic tumor cells". Cancer Chemother Pharmacol. 2006; 58:725-734. [PubMed: 16555088]

10. Gatza ML, Kung HN, Blackwell KL, Dewhirst MW, Marks JR, Chi JT. Analysis of tumor environmental response and oncogenic pathway activation identifies distinct basal and luminal features in HER2-related breast tumor subtypes. Breast Cancer Res. 2011; 13:R62. [PubMed: 21672245]

11. Yuneva M, Zamboni N, Oefner P, Sachidanandam R, Lazebnik Y. Deficiency in glutamine but not glucose induces MYC-dependent apoptosis in human cells. J Cell Biol. 2007; 178:93-105. [PubMed: 17606868]

12. Wise DR, DeBerardinis RJ, Mancuso A, Sayed N, Zhang XY, Pfeiffer HK, Nissim I, Daikhin E, Yudkoff M, McMahon SB, Thompson CB. Myc regulates a transcriptional program that stimulates mitochondrial glutaminolysis and leads to glutamine addiction. Proc Natl Acad Sci U S A. 2008; 105:18782-18787. [PubMed: 19033189]

13. Gao P, Tchernyshyov I, Chang TC, Lee YS, Kita K, Ochi T, Zeller KI, De Marzo AM, Van Eyk JE, Mendell JT, Dang CV. c-Myc suppression of miR-23a/b enhances mitochondrial glutaminase expression and glutamine metabolism. Nature. 2009; 458:762-765. [PubMed: 19219026]

14. Yang C, Sudderth J, Dang T, Bachoo RM, McDonald JG, DeBerardinis RJ. Glioblastoma cells require glutamate dehydrogenase to survive impairments of glucose metabolism or Akt signaling. Cancer Res. 2009; 69:7986-7993. [PubMed: 19826036]

15. Seltzer MJ, Bennett BD, Joshi AD, Gao P, Thomas AG, Ferraris DV, Tsukamoto T, Rojas CJ, Slusher BS, Rabinowitz JD, Dang CV, Riggins GJ. Inhibition of glutaminase preferentially slows growth of glioma cells with mutant IDH1. Cancer Res. 2010; 70:8981-8987. [PubMed: 21045145]

16. Kung HN, Marks JR, Chi JT. Glutamine synthetase is a genetic determinant of cell type-specific glutamine independence in breast epithelia. PLoS Genet. 2011; 7:e1002229. [PubMed: 21852960]

17. Sheen JH, Zoncu R, Kim D, Sabatini DM. Defective regulation of autophagy upon leucine deprivation reveals a targetable liability of human melanoma cells in vitro and in vivo. Cancer Cell. 2011; 19:613-628. [PubMed: 21575862]

18. Scott L, Lamb J, Smith S, Wheatley DN. Single amino acid (arginine) deprivation: rapid and selective death of cultured transformed and malignant cells. British journal of cancer. 2000; 83:800-810. [PubMed: 10952786] 
19. Kreis W, Baker A, Ryan V, Bertasso A. Effect of nutritional and enzymatic methionine deprivation upon human normal and malignant cells in tissue culture. Cancer Res. 1980; 40:634-641. [PubMed: 6937240]

20. Ohtawa K, Ueno T, Mitsui K, Kodera Y, Hiroto M, Matsushima A, Nishimura H, Inada Y. Apoptosis of leukemia cells induced by valine-deficient medium. Leukemia: official journal of the Leukemia Society of America, Leukemia Research Fund, UK. 1998; 12:1651-1652.

21. Terunuma A, Putluri N, Mishra P, Mathé EA, Dorsey TH, Yi M, Wallace TA, Issaq HJ, Zhou M, Killian JK, Stevenson HS, Karoly ED, Chan K, Samanta S, Prieto D, Hsu TYT, Kurley SJ, Putluri V, Sonavane R, Edelman DC, Wulff J, Starks AM, Yang Y, Kittles RA, Yfantis HG, Lee DH, Ioffe OB, Schiff R, Stephens RM, Meltzer PS, Veenstra TD, Westbrook TF, Sreekumar A, Ambs S. MYC-driven accumulation of 2-hydroxyglutarate is associated with breast cancer prognosis. The Journal of Clinical Investigation. 2014; 124:398-412. [PubMed: 24316975]

22. Timmerman LA, Holton T, Yuneva M, Louie RJ, Padro M, Daemen A, Hu M, Chan DA, Ethier SP, van 't Veer LJ, Polyak K, McCormick F, Gray JW. Glutamine Sensitivity Analysis Identifies the xCT Antiporter as a Common Triple-Negative Breast Tumor Therapeutic Target. Cancer Cell. 2013

23. Tang X, Keenan MM, Wu J, Lin CA, Dubois L, Thompson JW, Freedland SJ, Murphy SK, Chi JT. Comprehensive profiling of amino acid response uncovers unique methionine-deprived response dependent on intact creatine biosynthesis. PLoS genetics. 2015; 11:e1005158. [PubMed: 25849282]

24. Tang X, Wu J, Ding C-K, Lu M, Keenan MM, Lin C-C, Lin C-A, Wang CC, George D, Hsu DS, Chi J-T. Cystine deprivation triggers programmed necrosis in VHL-deficient renal cell carcinomas. Cancer Research. 2016

25. Neve RM, Chin K, Fridlyand J, Yeh J, Baehner FL, Fevr T, Clark L, Bayani N, Coppe JP, Tong F, Speed T, Spellman PT, DeVries S, Lapuk A, Wang NJ, Kuo WL, Stilwell JL, Pinkel D, Albertson DG, Waldman FM, McCormick F, Dickson RB, Johnson MD, Lippman M, Ethier S, Gazdar A, Gray JW. A collection of breast cancer cell lines for the study of functionally distinct cancer subtypes. Cancer Cell. 2006; 10:515-527. [PubMed: 17157791]

26. Murphy JM, Silke J. Ars Moriendi; the art of dying well - new insights into the molecular pathways of necroptotic cell death. EMBO reports. 2014; 15:155-164. [PubMed: 24469330]

27. Gatza ML, Lucas JE, Barry WT, Kim JW, Wang Q, Crawford MD, Datto MB, Kelley M, MatheyPrevot B, Potti A, Nevins JR. A pathway-based classification of human breast cancer. Proc Natl Acad Sci U S A. 2010; 107:6994-6999. [PubMed: 20335537]

28. Takekawa M, Posas F, Saito H. A human homolog of the yeast Ssk2/Ssk22 MAP kinase kinase kinases, MTK1, mediates stress-induced activation of the p38 and JNK pathways. EMBO J. 1997; 16:4973-4982. [PubMed: 9305639]

29. Arimoto K, Fukuda H, Imajoh-Ohmi S, Saito H, Takekawa M. Formation of stress granules inhibits apoptosis by suppressing stress-responsive MAPK pathways. Nat Cell Biol. 2008; 10:1324-1332. [PubMed: 18836437]

30. Chen JL, Merl D, Peterson CW, Wu J, Liu PY, Yin H, Muoio DM, Ayer DE, West M, Chi JT. Lactic acidosis triggers starvation response with paradoxical induction of TXNIP through MondoA. PLoS genetics. 2010; 6

31. Lucas JE, Kung HN, Chi JT. Latent factor analysis to discover pathway-associated putative segmental aneuploidies in human cancers. PLoS Comput Biol. 2010; 6:e1000920. [PubMed: 20824128]

32. Chin K, DeVries S, Fridlyand J, Spellman PT, Roydasgupta R, Kuo WL, Lapuk A, Neve RM, Qian Z, Ryder T, Chen F, Feiler H, Tokuyasu T, Kingsley C, Dairkee S, Meng Z, Chew K, Pinkel D, Jain A, Ljung BM, Esserman L, Albertson DG, Waldman FM, Gray JW. Genomic and transcriptional aberrations linked to breast cancer pathophysiologies. Cancer Cell. 2006; 10:529_ 541. [PubMed: 17157792]

33. Asiedu MK, Ingle JN, Behrens MD, Radisky DC, Knutson KL. TGFbeta/TNF(alpha)-mediated epithelial-mesenchymal transition generates breast cancer stem cells with a claudin-low phenotype. Cancer Res. 2011; 71:4707-4719. [PubMed: 21555371] 
34. Ho M-Y, Tang S-J, Chuang M-J, Cha T-L, Li J-Y, Sun G-H, Sun K-H. TNF-a Induces EpithelialMesenchymal Transition of Renal Cell Carcinoma Cells via a GSK3 $\beta$-Dependent Mechanism. Molecular Cancer Research. 2012; 10:1109-1119. [PubMed: 22707636]

35. Harten SK, Shukla D, Barod R, Hergovich A, Balda MS, Matter K, Esteban MA, Maxwell PH. Regulation of renal epithelial tight junctions by the von Hippel-Lindau tumor suppressor gene involves occludin and claudin 1 and is independent of E-cadherin. Mol Biol Cell. 2009; 20:10891101. [PubMed: 19073886]

36. Zhao W, Prijic S, Urban BC, Tisza MJ, Zuo Y, Li L, Tan Z, Chen X, Mani SA, Chang JT. Candidate anti-metastasis drugs suppress the metastatic capacity of breast cancer cells by reducing membrane fluidity. Cancer Res. 2016

37. Li CW, Xia W, Huo L, Lim SO, Wu Y, Hsu JL, Chao CH, Yamaguchi H, Yang NK, Ding Q, Wang Y, Lai YJ, LaBaff AM, Wu TJ, Lin BR, Yang MH, Hortobagyi GN, Hung MC. Epithelialmesenchymal transition induced by TNF-alpha requires NF-kappaB-mediated transcriptional upregulation of Twist1. Cancer Res. 2012; 72:1290-1300. [PubMed: 22253230]

38. Zhang X, Zhao X, Shao S, Zuo X, Ning Q, Luo M, Gu S, Zhao X. Notch1 induces epithelialmesenchymal transition and the cancer stem cell phenotype in breast cancer cells and STAT3 plays a key role. Int J Oncol. 2015; 46:1141-1148. [PubMed: 25544568]

39. Kulbe H, Thompson R, Wilson JL, Robinson S, Hagemann T, Fatah R, Gould D, Ayhan A, Balkwill $\mathrm{F}$. The inflammatory cytokine tumor necrosis factor-alpha generates an autocrine tumorpromoting network in epithelial ovarian cancer cells. Cancer Res. 2007; 67:585-592. [PubMed: 17234767]

40. Wu Y, Zhou BP. TNF-alpha/NF-kappaB/Snail pathway in cancer cell migration and invasion. British journal of cancer. 2010; 102:639-644. [PubMed: 20087353]

41. Paranjape AN, Soundararajan R, Werden SJ, Joseph R, Taube JH, Liu H, Rodriguez-Canales J, Sphyris N, Wistuba I, Miura N, Dhillon J, Mahajan N, Mahajan K, Chang JT, Ittmann M, Maity SN, Logothetis C, Tang DG, Mani SA. Inhibition of FOXC2 restores epithelial phenotype and drug sensitivity in prostate cancer cells with stem-cell properties. Oncogene. 2016

42. Yae T, Tsuchihashi K, Ishimoto T, Motohara T, Yoshikawa M, Yoshida GJ, Wada T, Masuko T, Mogushi K, Tanaka H, Osawa T, Kanki Y, Minami T, Aburatani H, Ohmura M, Kubo A, Suematsu M, Takahashi K, Saya H, Nagano O. Alternative splicing of CD44 mRNA by ESRP1 enhances lung colonization of metastatic cancer cell. Nat Commun. 2012; 3:883. [PubMed: 22673910]

43. Ishimoto T, Nagano O, Yae T, Tamada M, Motohara T, Oshima H, Oshima M, Ikeda T, Asaba R, Yagi H, Masuko T, Shimizu T, Ishikawa T, Kai K, Takahashi E, Imamura Y, Baba Y, Ohmura M, Suematsu M, Baba H, Saya H. CD44 variant regulates redox status in cancer cells by stabilizing the XCT subunit of system xc(-) and thereby promotes tumor growth. Cancer Cell. 2011; 19:387400. [PubMed: 21397861]

44. Motzer RJ, Hutson TE, Cella D, Reeves J, Hawkins R, Guo J, Nathan P, Staehler M, de Souza P, Merchan JR, Boleti E, Fife K, Jin J, Jones R, Uemura H, De Giorgi U, Harmenberg U, Wang J, Sternberg CN, Deen K, McCann L, Hackshaw MD, Crescenzo R, Pandite LN, Choueiri TK. Pazopanib versus sunitinib in metastatic renal-cell carcinoma. N Engl J Med. 2013; 369:722-731. [PubMed: 23964934]

45. Foulkes WD, Smith IE, Reis-Filho JS. Triple-negative breast cancer. N Engl J Med. 2010; 363:1938-1948. [PubMed: 21067385]

46. Yoshikawa M, Tsuchihashi K, Ishimoto T, Yae T, Motohara T, Sugihara E, Onishi N, Masuko T, Yoshizawa K, Kawashiri S, Mukai M, Asoda S, Kawana H, Nakagawa T, Saya H, Nagano O. xCT inhibition depletes CD44v-expressing tumor cells that are resistant to EGFR-targeted therapy in head and neck squamous cell carcinoma. Cancer Res. 2013; 73:1855-1866. [PubMed: 23319806]

47. Godwin AK, Meister A, O'Dwyer PJ, Huang CS, Hamilton TC, Anderson ME. High resistance to cisplatin in human ovarian cancer cell lines is associated with marked increase of glutathione synthesis. Proc Natl Acad Sci U S A. 1992; 89:3070-3074. [PubMed: 1348364]

48. Moore T, Le A, Niemi AK, Kwan T, Cusmano-Ozog K, Enns GM, Cowan TM. A new LC-MS/MS method for the clinical determination of reduced and oxidized glutathione from whole blood. $\mathrm{J}$ Chromatogr B Analyt Technol Biomed Life Sci. 2013; 929:51-55.

49. Keenan MM, Liu B, Tang X, Wu J, Cyr D, Stevens RD, Ilkayeva O, Huang Z, Tollini LA, Murphy SK, Lucas J, Muoio DM, Kim SY, Chi JT. ACLY and ACC1 Regulate Hypoxia-Induced Apoptosis 
by Modulating ETV4 via alpha-ketoglutarate. PLoS Genet. 2015; 11:e1005599. [PubMed: 26452058] 
A.

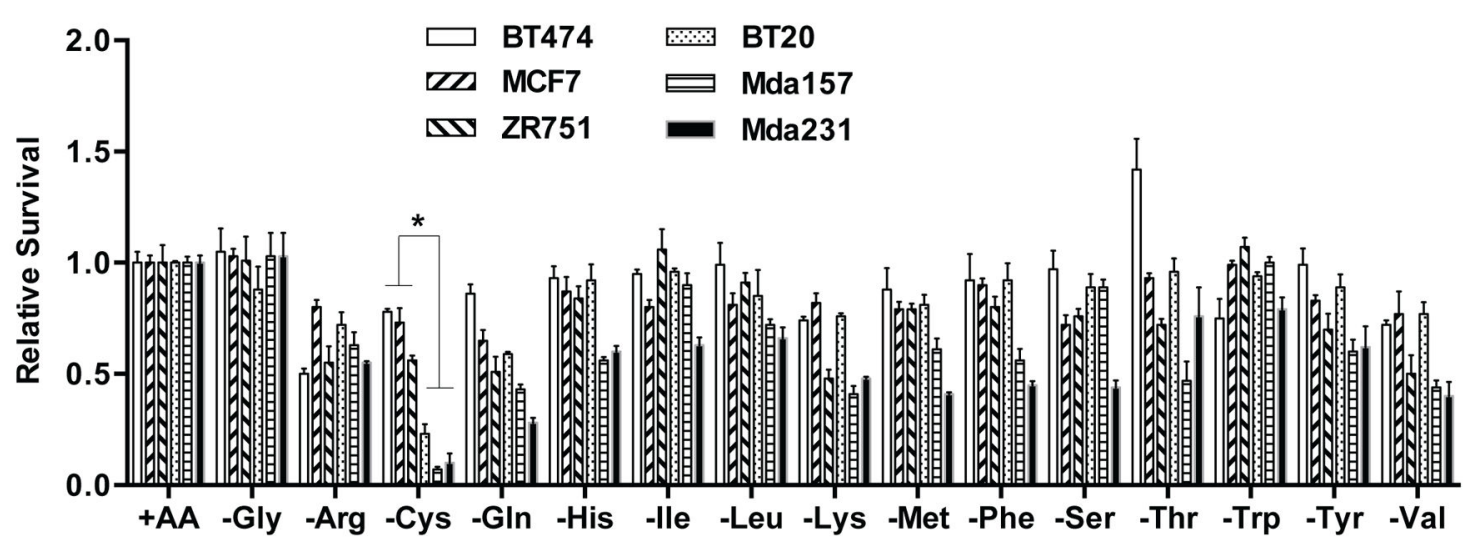

B.

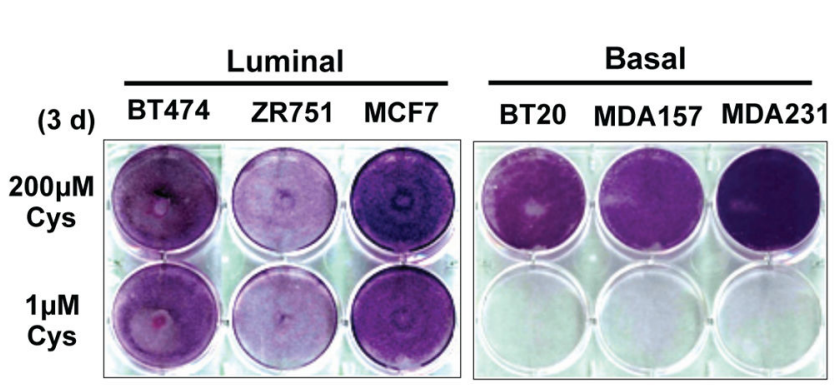

C.

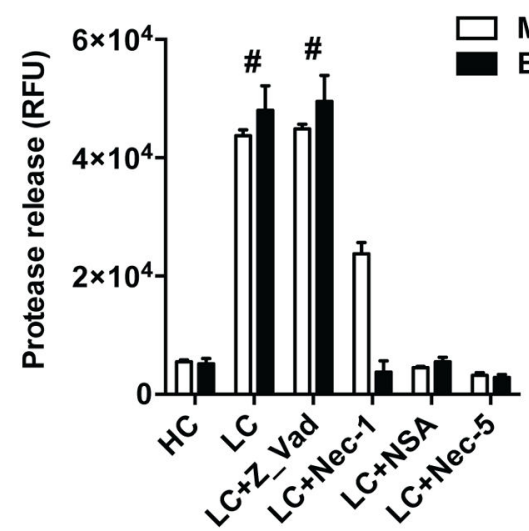

Figure 1. Nutrigenetic screens identified cystine addiction of basal breast cancer cells (A). Cell viability of a panel of breast cancer cells determined by CellTiter-Glo® Assay after deprivation of indicated amino acid for 48 hours $(n=3, p<0.01)$. Mda157: MDA-MB-157;

Mda231: MDA-MB-231

(B). Survival of indicated breast cancer cells stained by crystal violet after exposure to regular $(200 \mu \mathrm{M})$ or low $(1 \mu \mathrm{M})$ levels of cystine.

(C). Cell cytotoxicity of MDA-MB-231 and BT549 cells was determined by protease release $(\mathrm{n}=3, \mathrm{p}<0.001)$ under high $(\mathrm{HC}, 200 \mu \mathrm{M})$ or low cystine $(\mathrm{LC}, 1 \mu \mathrm{M})$ treated with $\mathrm{z}$-VadFMK $(20 \mu \mathrm{M})$, Nec-1 $(10 \mu \mathrm{M})$, NSA $(5 \mu \mathrm{M})$ or Nec-5 $(5 \mu \mathrm{M})$. 
A.

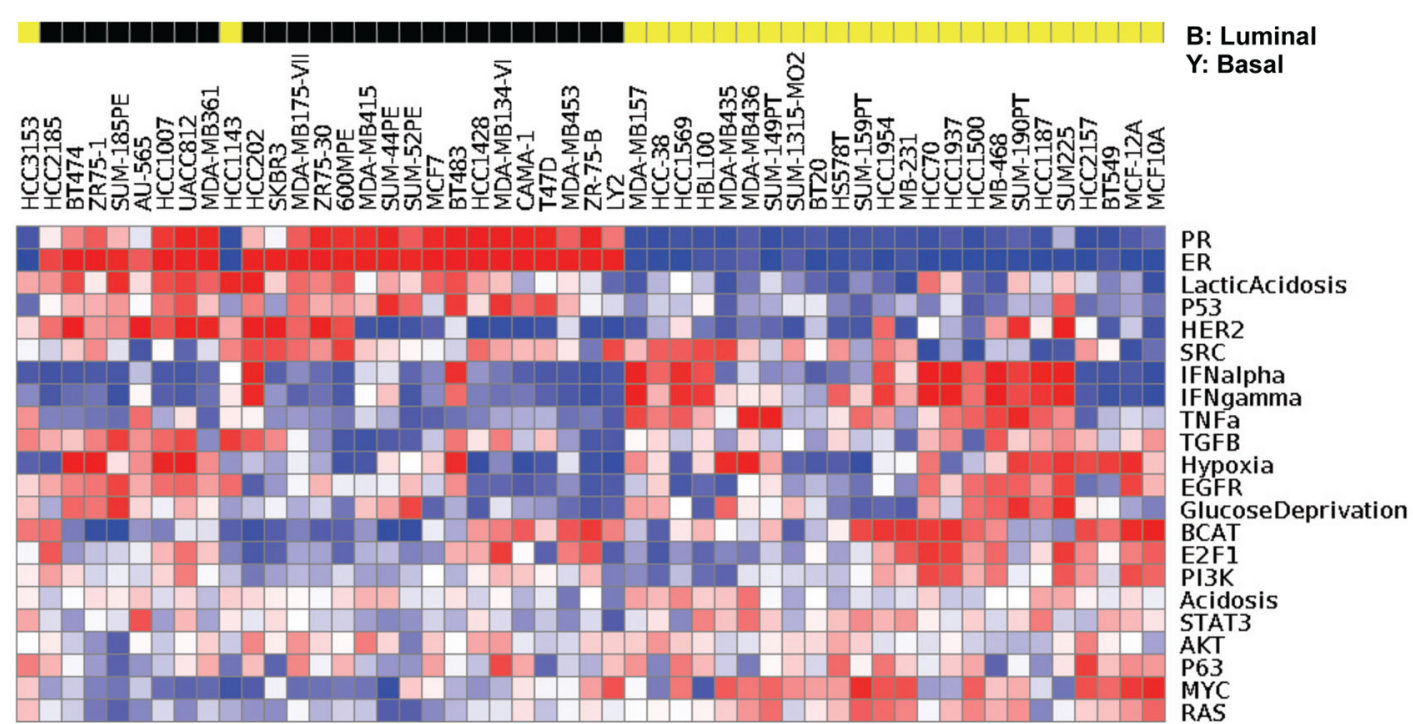

B. MDA231

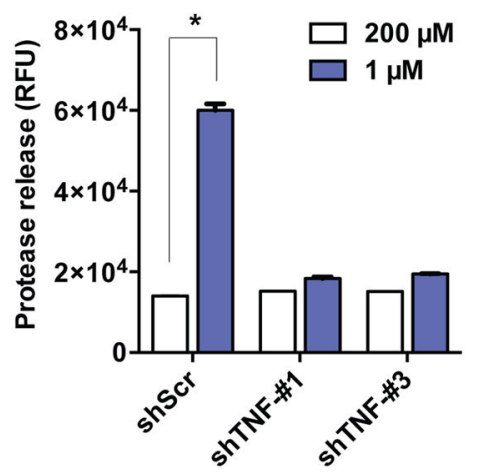

C.

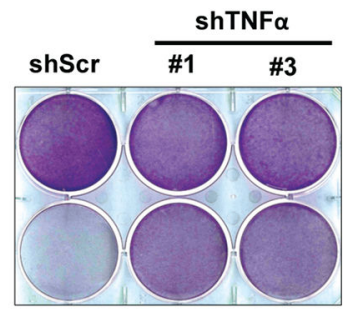

D.

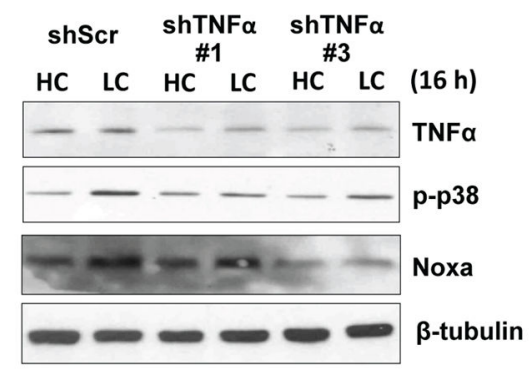

Figure 2. Increased TNFa activity is required for cystine-deprived necrosis in basal-type breast cancer cells

(A). The activities of various pathways were estimated in 51 human breast cancer cell lines based on their expression data (E-TABM-157).

(B, C, D). Cell cytotoxicity (B; n=3, p< 0.01), viability (C), and analysis of TNFa, p-p38 and Noxa protein (D) of MDA-MB-231 shScr and two shTNFa cells under indicated levels of cystine. 
A.

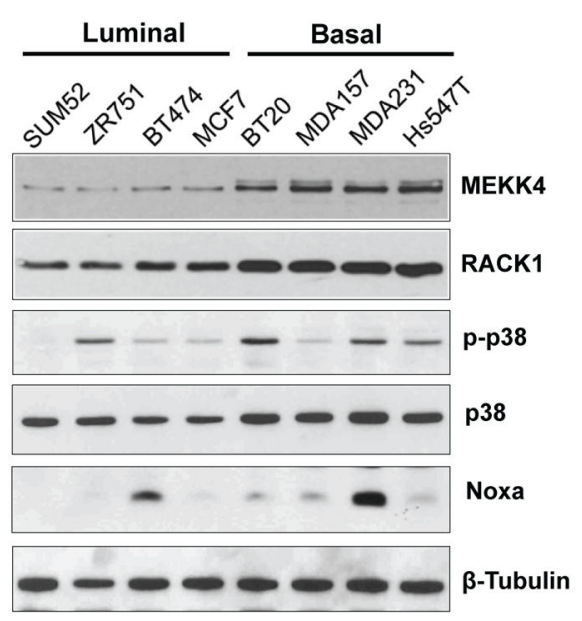

B. BT549

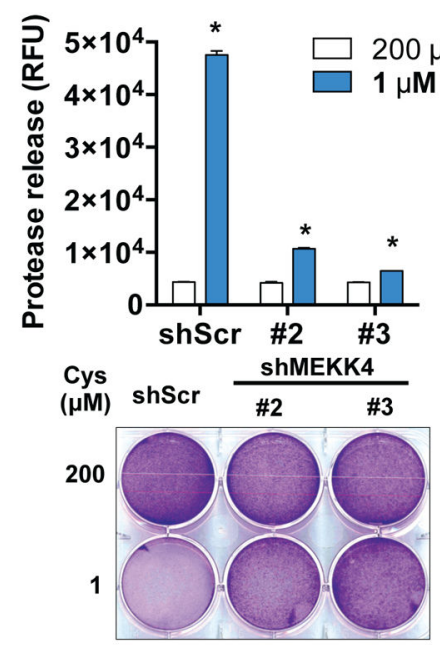

C.

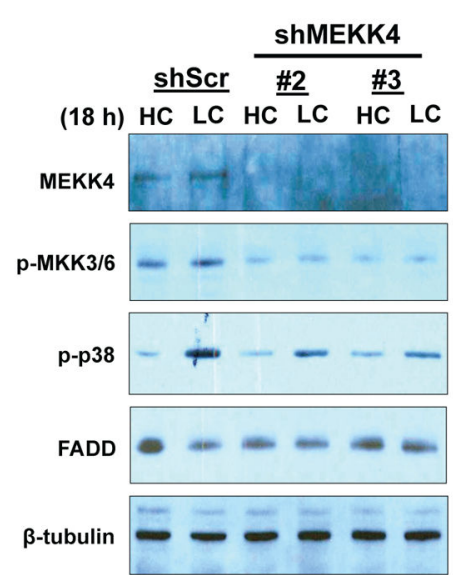

Figure 3. Enhanced MEKK4-p38-Noxa pathway in basal-type breast cancer cells contributes to cystine-deprived necrosis

(A). Immunoblotting of indicated proteins in a panel of luminal or basal type breast cancer cells.

(B). Cell cytotoxicity and survival of BT549 infected with shScr and two different shMEKK4 under indicated levels of cystine for 24 hours $(n=4, p<0.01)$.

(C). Immunoblotting of MEKK4, MKK3/6 phosphorylation and p38 phosphorylation in BT549 with shScr and shMEKK4 under high $(\mathrm{HC}, 200 \mu \mathrm{M})$ or low cystine $(\mathrm{LC}, 1 \mu \mathrm{M})$ for 18 hours. 

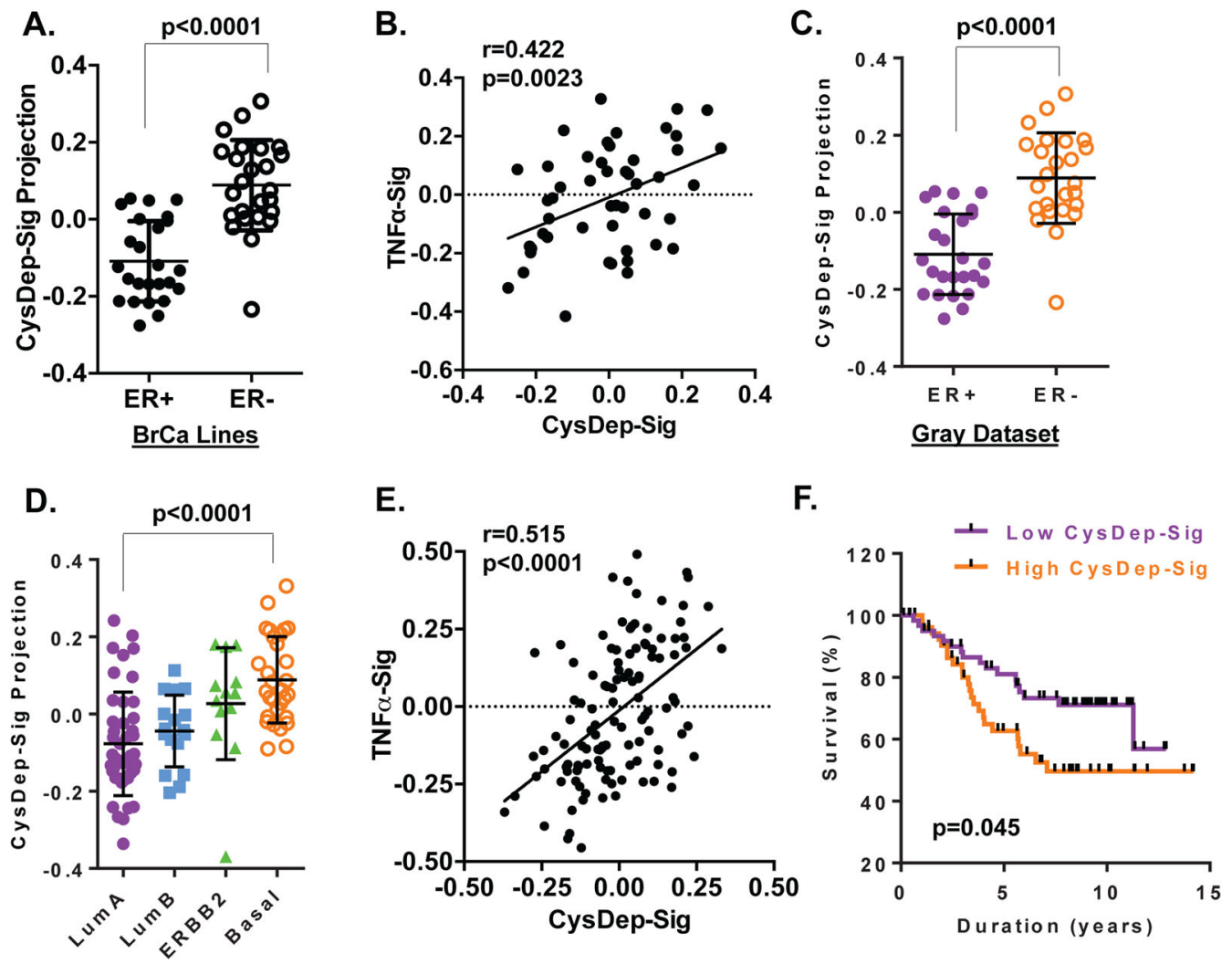

Figure 4. Enrichment and correlation of CysDep and TNFa gene signatures in breast tumors and cancer cell lines

(A). The activities of CysDep gene signature in a dataset of breast cancer cell lines in relation to ER status.

(B). The correlation of CysDep and TNFa gene signatures in a panel of breast cancer cell lines.

(C, D). The activities of CysDep gene signature in a dataset of breast cancer relative to ER status (C) or intrinsic subtypes (D).

(E). The correlations of CysDep and TNFa gene signature in a panel of breast tumor datasets.

(F). Kaplan-Meyer survival curves of high and low CysDep-Sig breast tumors ${ }^{32}$. 
A. MDA231

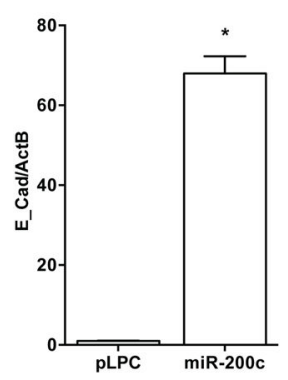

B.

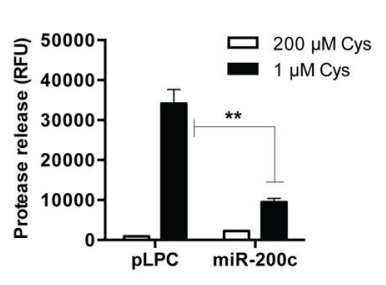

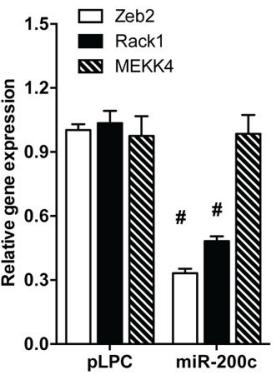

c.

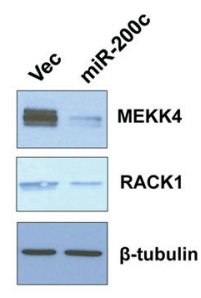

D.

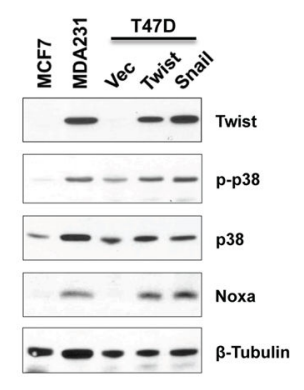

E.

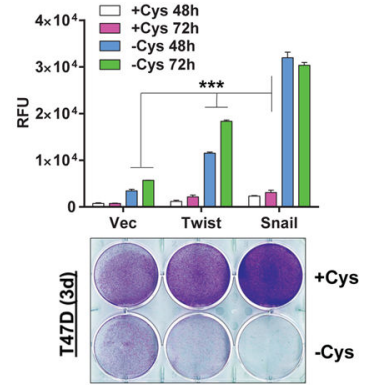

Figure 5. Control of the cystine addiction phenotype by EMT regulators

(A). The mRNA expression of indicated genes in MDA-MB-231 transfected with pLPC (vector) or miR-200c $(\mathrm{n}=3$; *, $\mathrm{p}<0.001 ; \#, \mathrm{p}<0.05)$.

(B) Analysis MEKK4 and RACK1 protein expression in MDA-MB-231 transfected with $\mathrm{pLPC}$ (vector) and miR-200c cells $(\mathrm{n}=3, \mathrm{p}<0.01)$.

(C) Cell cytotoxicity (protease release) of either MDA-MB-231 pLPC (vector) or miR-200c cells under high (HC, $200 \mu \mathrm{M})$ or low cystine (LC, $1 \mu \mathrm{M})$ for 24 hours.

(D). Immunoblots of Twist, MEKK4, p-p38 and Noxa protein expression in luminal-type T47D cells infected with either Vec (pWZL), Twist or Snail.

(E). Cell cytotoxicity (protease release; $n=3, p<0.05$ ) and survival (crystal violet staining) of T47D Vec, Twist and Snail cells under cystine deprivation for 2 or 3 days. 


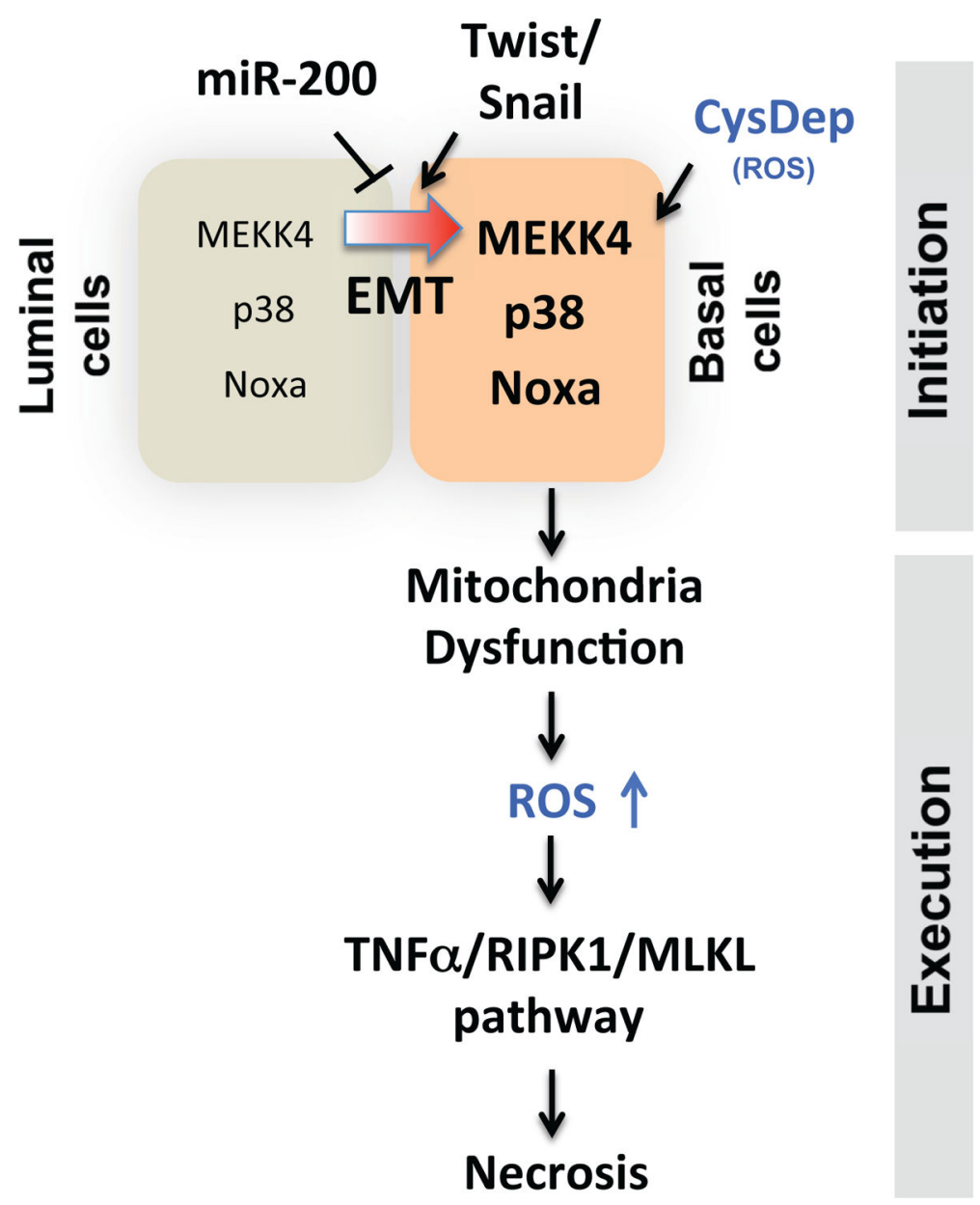

Figure 6.

The model of EMT mediated cystine-deprived necrosis of breast cancer cells. 